\title{
Experience matters: A psycholinguistic investigation of predicates of personal taste*
}

\author{
Elsi Kaiser \\ University of Southern California
}

\author{
Jamie Herron Lee \\ University of Southern California
}

\begin{abstract}
We discuss two psycholinguistic experiments on subjective adjectives, with the aim of testing the idea that predicates of personal taste (PPTs, e.g. fun, tasty) and non-PPT multidimensional adjectives (e.g. smart, healthy) differ in terms of the perspective-taking they involve. Although both are widely regarded as involving a judge/evaluator, it has recently been proposed that the judge of a PPT needs to be an experiencer, while judges of multidimensional non-PPT adjectives do not have this requirement. We report a new study investigating this claim that builds on and extends our earlier work, by using sentences with one animate and one inanimate argument. This allows us to avoid potential complications from pronoun ambiguity. We manipulated verb argument structure (Experiencer-Theme vs. Agent-Patient verbs) to test if PPTs prefer experiencer judges. The results confirm that PPTs, but not non-PPT multidimensional adjectives, are sensitive to the presence of an experiencer thematic role.
\end{abstract}

Keywords: predicates of personal taste, subjectivity, multidimensional adjectives, perspective-taking, judge, attitude holder, experiencer

\section{Introduction}

The notion of evaluation is fundamental to human cognition and perception (e.g. Jarvis \& Petty 1996). A person's evaluative attitudes are highly subjective, as they are linked to a particular individual's traits, knowledge and experiences. Given how central evaluative attitudes are to cognition, it is not surprising that there exists a range of linguistic expressions - e.g. predicates of personal taste (ex.1a) and epithets (ex.1b) - that reflect subjective attitudes. These subjective expressions involve individuals' attitudes, thoughts and feelings, and to fully understand them, one needs to know whose judgment, opinion, or knowledge state is being conveyed. Who finds salmon tasty? Who thinks that Bob is an idiot? These expressions have been analyzed as making reference to a 'judge' or

\footnotetext{
${ }^{*}$ We would like to thank the SALT 27 audience for useful comments and feedback.
}

(C) 2017 Kaiser and Herron Lee 
'evaluator' (e.g. Lasersohn 2005; Potts 2007; Stephenson 2007; Patel-Grosz 2012 and many others, but see also Pearson 2013). Much of the research on these expressions builds on Lasersohn's (2005) judge parameter $j$, such that the truth of sentences containing predicates of personal taste is relativized to the particular individual who is the judge.

\section{(1) a. Salmon is tasty.}

b. Bob missed the train again! The idiot forgot to set his alarm last night.

In addition to the specific class of adjectives known as predicates of personal taste, the general class of multidimensional adjectives (e.g. Kamp 1975; Klein 1980; Sassoon 2013; Kennedy 2013; Stojanovic \& McNally 2017) is also commonly viewed as involving subjectivity. Adjective such as healthy, ugly, beautiful and stupid are multidimensional because they involve multiple criteria that can be used for ranking/ordering individuals with the property. For example, with 'healthy', one could consider the cardiovascular system and/or the immune system when assessing someone's health.

Multidimensional adjectives contrast with unidimensional adjectives (e.g. tall, old), for which "exactly one criterion is used to order individuals according to the property [they] describe" (McNally \& Stojanovic 2017: 20). With multidimensional adjectives, subjectivity stems from the fact that individuals can disagree regarding the significance of each dimension (McNally \& Stojanovic 2017, see also Kennedy 2013; Bylinina 2014). For example, is Amy healthier than Bill if she has a severe immune disorder that is controlled by medication, but he has a mild heart condition that sometimes impacts his daily life? Two people could reasonably disagree about this. With regard to unidimensional adjectives, individuals can disagree about the threshold/cut-off point (e.g. how old does someone have to be to count as 'old'?) but people's judgments about the relative ordering of individuals with respect to the particular property (e.g. relative oldness of individual $\mathrm{X}$ compared to individual $\mathrm{Y}$ ) are not expected to differ (see McNally \& Stojanovic 2017 for discussion).

Some adjectives which are normally classified as predicates of personal taste (PPTs) can arguably also be multidimensional. For example, one could say, 'Bob is interesting, except for his taste in music which is very mainstream' - in other words, while some aspects/dimensions of Bob are interesting (to the speaker), others are not. (See Sassoon 2013 on using 'except' and 'in \{every/some\} \{respect/way\}' to test whether an adjective is unidimensional or multidimensional). However, not all multidimensional adjectives are PPTs.

In this paper, as the subsequent sections will make clear, we focus on investigating the potential differences between PPT adjectives and non-PPT multidimensional adjectives, in order to better understand the class of subjective predicates of personal taste. 
A psycholinguistic investigation of predicates of personal taste

\subsection{The contribution of experience to the meaning of subjective adjectives}

Recently, it has been claimed that there is a fundamental distinction between PPTs and non-PPT multidimensional adjectives, namely that (a) PPTs crucially involve an experiencer argument, whereas (b) multidimensional adjectives that are not PPTs (e.g. smart, healthy) do not have an experiencer argument (e.g. Bylinina 2014; McNally \& Stojanovic 2017). In essence, for something to be 'fun' or 'tasty', someone must have the relevant experience (usually the speaker), but for something/someone to be 'stupid' or 'healthy', no such experiencing is necessary. McNally \& Stojanovic (2017: 24) mention “...the presence (or absence) of an experiencer, that is, a sentient individual who perceives the property in question..." and argue for the importance of distinguishing "predicates that entail a proper experiencer from those whose subjective element may be due to differences of opinion over where the threshold for ascribing a property lies or what the relative weights of the different criteria for ascribing it should be" (McNally \& Stojanovic 2017: 28) - in other words, they argue for a distinction between subjective predicates that entail an experiencer (PPTs) and predicates whose subjectivity stems from disagreement regarding thresholds or dimension weights (unidimensional or multidimensional adjectives).

The significance of a sentient experiencer is also discussed by Bylinina (2014), who similarly argues that the "presence or absence of an experiencer argument is orthogonal to judge-dependence" (Bylinina 2014: 50). She proposes a distinct 'judge $=$ experiencer' requirement, namely that "A direct statement about someone's internal state can be made only if the judge parameter is set to the same value as the experiencer of this internal state" (Bylinina 2014: 58). She argues that this requirement applies to PPTs but is not limited only to them. In her semantics for tasty (ex.2), the second part states that the judge $j$ and experiencer $z$ refer to the same individual. Bylinina distinguishes adjectives like tasty that refer to experience from adjectives like smart/healthy which she argues do not refer to experience in the same way.

$\llbracket \operatorname{tasty} \rrbracket^{c ; w, t, \mathbf{j}}=$

(i) $\lambda z \lambda x . \exists s\left[\operatorname{taste}(s) \& \operatorname{Experiencer}(s, z) \& \operatorname{Stimulus}(s, x) \& \operatorname{TASTE}(s)>d_{\mathrm{st}}\right.$ for $j$ at $t$ in $w$ ];

(ii) JUDGE=EXPERIENCER: $j=z$

Indeed, the significance of experience and the judge/experiencer relation has been explored in depth in recent work (e.g. Kennedy \& Willer 2016; Sæbø 2009; Pearson 2013; Ninan 2014). However, intuitions regarding the role and even the presence of experiencers can be murky (e.g. see McNally \& Stojanovic's detailed 
discussion of aesthetic predicates like 'beautiful' and 'ugly'). Suggested tests for identifying adjectives with judges and/or experiencers - e.g. evaluative use of the find construction - have generated debate and do not always yield a reliable diagnostic (see e.g. McNally \& Stojanovic 2017; Sæbø 2009; Kennedy 2013; Bylinina 2014: 34 for discussion). In our work, we explore the possibility of probing the contribution of experiencers by means of verb argument structure, as discussed in Sections 2 and 3.

\section{Aims of the current experiment and its relation to our prior work}

The experiment reported in the current paper explores the general question of how to identify adjectives with experiencers from a psycholinguistic perspective, in order to further our understanding of the proposed distinction between subjective predicates that entail experiences (PPTs) and those that do not (e.g. non-PPT multidimensional adjectives). We aim to experimentally test whether PPTs show a sensitivity to the presence of experiencers that merely multidimensional nonPPT adjectives do not. To do this, we manipulate the availability of an experiencer argument in the immediately preceding discourse by means of verb argument structure. Specifically, we compare contexts with verbs that introduce experiencer arguments in subject position (Experiencer-Theme verbs, e.g. heard, understood, looked at, Ambridge at al. 2016) to contexts with verbs that do not have experiencer subjects (Agent-Patient verbs, e.g. nudged, kicked). When comprehenders encounter a PPT or a non-PPT multidimensional adjective (after a sentence with an experiencer-introducing or non-experiencer introducing verb), we can test whether they show a preference to interpret the recently-introduced experiencer as the judge of the subjective adjective and whether this depends on the type of adjective.

The present experiment builds on an earlier study that we conducted on this same topic, but extends it in certain key respects (discussed in Section 3). In the current section, we review our first experiment, as it provides the backdrop for the new study presented in this paper.

\subsection{Earlier study: Design, materials, method, participants}

To test whether people's interpretation of the judge/evaluator of PPTs and nonPPT multidimensional adjectives is influenced by the presence/absence of an experiencer, introduced by a verb in the preceding discourse, we (Kaiser \& Herron Lee to appear) conducted an initial questionnaire study that manipulated the nature of the adjective in the second sentence (PPT vs. non-PPT multidimensional adjective, ex.4) and the verb in the first sentence (ExperiencerTheme vs. Agent-Patient, ex.3, abbreviated as ExpThe and AgPat respectively). 
A psycholinguistic investigation of predicates of personal taste

Ex.(3-5) provides an example item.

The various PPTs and non-PPT multidimensional adjectives ${ }^{1}$ were selected on the basis of prior work (e.g. Pearson 2013; Bylinina 2014; McNally \& Stojanovic 2017). The Experiencer-Theme verbs were selected based on Ambridge et al. (2016) and the Agent-Patient verbs based on criteria from other published work. Experiencer-Theme verbs have an experiencer subject (e.g. perception verbs like hear and conceptual verbs like understand, all from Ambridge at el.'s 2016 set of Experiencer-Theme verbs), unlike Agent-Patient verbs (e.g. push, carry, poke). These two verb classes allowed us to manipulate whether the preceding context contains an experiencer argument that can potentially fulfill the role of judge.

(3) a. First sentence (Experiencer-Theme verb): Jennifer looked at Amy.

b. First sentence (Agent-Patient verb): Jennifer nudged Amy.

a. Second sentence: She was irritating. [Predicate of personal taste]

b. Second sentence: She was smart. [Multidimensional adjective]

(5) Whose opinion is it that the other person is \{irritating/smart\}?

Jennifer / Amy / Narrator

As discussed in Kaiser \& Herron Lee (to appear), we decided to use Experiencer-Theme verbs rather than Stimulus-Experiencer verbs (e.g. frighten, worry, amuse), to avoid a possible confound stemming from pronoun interpretation effects: Because (i) pronouns are known to have a general bias for referring to preceding subjects (e.g. Chafe 1976; Crawley \& Stevenson 1990) and (ii) our stimuli were designed so that the pronoun refers to the non-judge referent, had we used Stimulus-Experiencer verbs, we might have obtained an overall preference for interpreting the object experiencer as the judge simply due to the preference to interpret pronouns as referring to subjects. In other words, if we had used object-experiencer verbs (such as the class of Stimulus-Experiencer verbs), then a finding that the preceding experiencer object tends to be interpreted as the judge could have been attributed to pronouns preferring subject antecedents, rather than having to do with the adjective entailing a judge that is an experiencer. Thus, Experiencer-Theme verbs (with subject-position experiencers) were a better choice (see Kaiser \& Herron Lee to appear for further discussion).

Native English speaking adults $(n=60)$ read excerpts (ex.3-4) that they were told are from stories and were asked to imagine they were reading extracts from novels. ${ }^{2}$ They answered questions (ex.5) that were designed to probe who they

\footnotetext{
${ }^{1}$ We also tested non-judge-dependent nongradable adjectives in another phase of the study, but the 'whose opinion' question is not applicable to non-judge-dependent expressions.

2 Earlier work (Kaiser 2015) found that processing of perspective-sensitive and subjective expressions can be modulated by the genre of the text (e.g. fiction vs. emails). This is presumably due to the existence of a narrator in fiction (and the fact that characters in fiction can act as judges)
} 
interpreted as the judge/evaluator of the subjective adjective. To answer these 'whose opinion' questions, participants had to choose one of the three answer choices (preceding subject, object, or narrator).

Crucially, all critical items in this study contained ambiguous pronouns. For example, in (4), 'she' could in principle refer to either Jennifer or Amy. This was done deliberately: We wanted to ensure that the judge of the opinion expressed in the critical second sentence would be as ambiguous as possible - for example, in ex.(4) it could be Jennifer's, Amy's or the narrator's opinion. The ambiguity of the pronoun has the advantage of making the critical question ('Who thinks the other person is irritating/smart?') maximally open-ended and unbiasing. ${ }^{3}$

\subsection{Predictions}

We assumed that Experiencer-Theme verbs would provide an available experiencer argument for a subsequent adjective seeking an experiencer judge, whereas Agent-Patient verbs would not. Thus, we hypothesized that if PPTs entail an experiencer judge but non-PPT multidimensional adjectives do not, participants' interpretations of the judge's identity would be influenced by verb type in the case of PPTs, but not non-PPT multidimensional adjectives: In the PPT conditions, the rate of subject-opinion answers to the 'whose opinion' question (ex.6) should be higher with Experiencer-Theme than Agent-Patient verbs. In non-PPT multidimensional adjectives conditions, both verb types are expected to result in similar proportions of subject-opinion responses.

\subsection{Results of our earlier study}

Figure 1 shows the proportion of subject's opinion, object's opinion and narrator's opinion choices in response to the question 'Whose opinion is it that the other person is [ADJECTIVE]?'. Statistical analyses (using logistic mixed effects regression) of the proportion of subject responses show that with PPTs, Experiencer-Theme verbs result in a significantly higher rate of subject-opinion responses than Agent-Patient verbs. With non-PPT multidimensional adjectives, both verb types yield comparable rates of subject-opinion responses that do not

vs. a more direct communicative context such as a conversation or an email where the speaker tends to be the default judge. Thus, both our earlier study and the new study presented in this paper were designed so that the stimuli were clearly presented as excerpts from fictional narratives.

3 We also probed people's interpretation of the ambiguous pronoun. The results are in line with the conclusions we draw from the explicit 'whose opinion' question data for PPTs and non-PPT multidimensionals. (See also Kaiser 2015 for earlier work on a related topic using a similar method.) 
A psycholinguistic investigation of predicates of personal taste

differ significantly (see Kaiser \& Herron Lee to appear for details). As predicted, verb type matters with PPTs (which prefer experiencer judges) but has no significant effect in the case of multidimensional non-PPT adjectives.

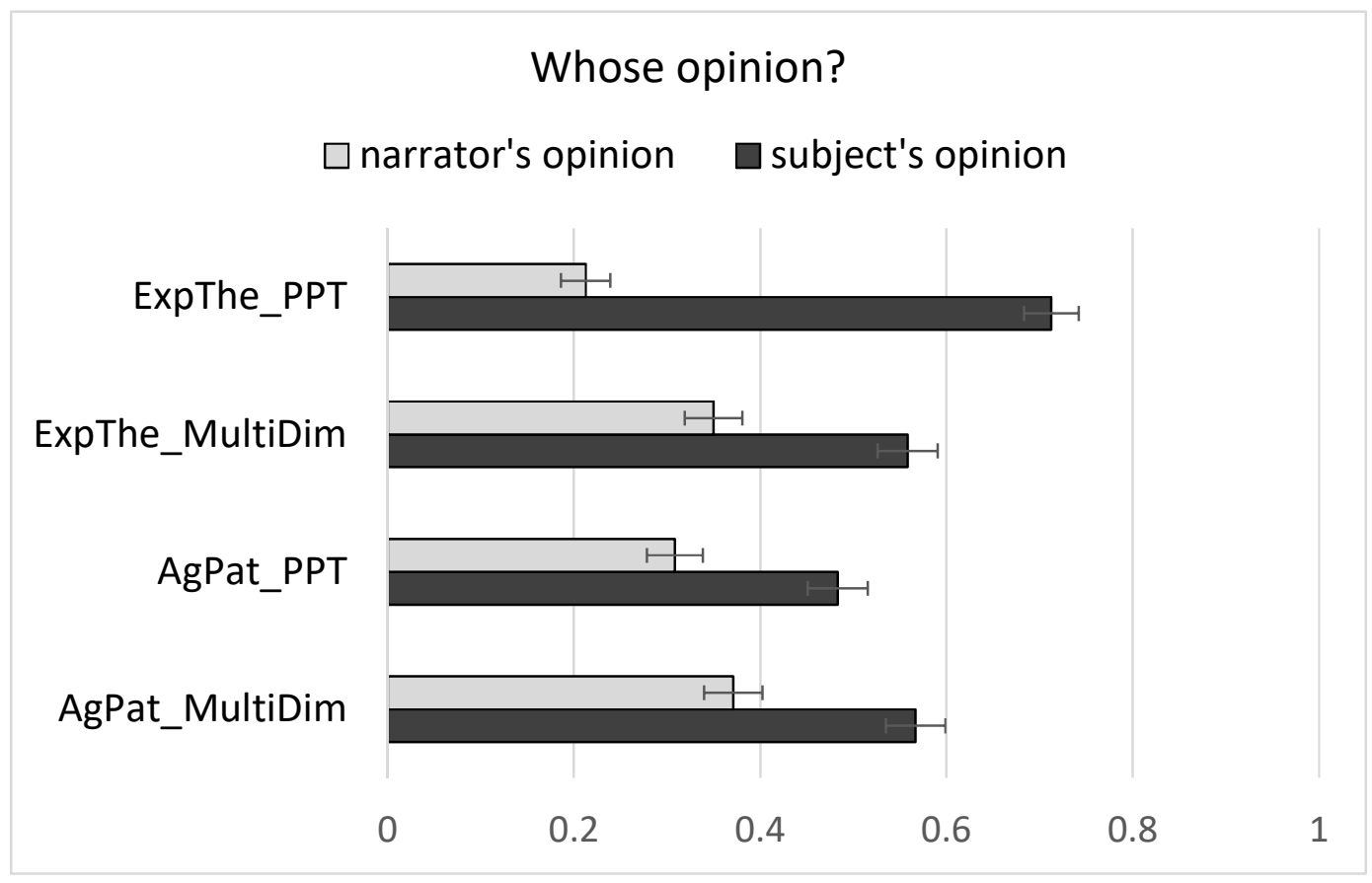

Figure 1 The proportion of 'subject's opinion' and 'narrator's opinion' responses to the question 'Whose opinion is it that the other person is [adjective]? ('Object's opinion' responses are not shown here, see Kaiser \& Herron Lee to appear for more details.) Error bars show +/$1 \mathrm{SE}$.

\section{Current study}

As seen in Section 2, our earlier study found that predicates of personal taste - but not non-PPT multidimensional adjectives - have a significant preference for judges who are experiencers (Kaiser \& Herron Lee to appear). In that study, we intentionally used ambiguous pronouns in the critical sentence (e.g. 'She was irritating/smart'), to ask the 'whose perspective' question in a maximally unbiased way. Given that the preceding sentence contains two same-gender referents, the 
pronoun can refer to the preceding subject or object, and the attitude-holder could be the preceding subject, preceding object or the narrator.

However, the use of ambiguous pronouns also brings potential complications. In particular, what if independent biases regarding pronoun interpretation influence how people resolve the pronoun, and this consequently influences who participants interpret as the judge? Our stimuli (e.g. Jennifer looked at Amy. She was irritating) were designed so that the referent of the pronoun (whether it be Jennifer or Amy) is not the judge. If I interpret 'she' as referring to Jennifer, Amy is the judge: Amy is irritated by Jennifer looking at her. Conversely, if I interpret 'she' as referring to Amy, Jennifer is the judge: Jennifer looked at Amy because Amy is irritating her. (We discuss coherence relations in Section 5.) If the narrator is the judge, the pronoun could, presumably, refer to either the subject or the object character. Importantly, for this earlier study, we tried to minimize potential referential biases by creating targets where the pronoun could, in principle, refer to either the subject or the object. Furthermore, we avoided Stimulus-Experiencer verbs which could have created an artificial experiencer preference due to the well-known bias for pronouns to prefer subject antecedents.

However, there is still the possibility that our results may be (partially) driven by pronoun interpretation biases, simply because the interpretation of who the pronoun refers to is partially yoked to the interpretation of who the judge is.

Thus, the current study has two main aims: First, to avoid potential complications stemming from pronoun ambiguity and to get a clearer picture of whether the experiencer/non-experiencer status of a referent influences its likelihood of being interpreted as the judge, we used items in which the first sentence has an animate subject and inanimate object and the second sentence uses the pronoun 'it' (ex.6-7). Thus, the pronoun is no longer ambiguous between the preceding subject and object. (Event reference is ruled out, as explained below.) Second, the current study also provides a near-replication of our earlier experiment, as we manipulate the same factors: (i) adjective type (PPT vs. multidimensional) and (ii) verb type (Agent-Patient vs. Theme-Experiencer). Recently, the importance and benefits of replication has received increasing attention (e.g. Open Science Collaboration 2015, The Reproducibility Project: Psychology, https://osf.io/ezcuj/wiki/home/). Showing that the same basic pattern of results obtains (in our case, in a slightly different context) is important for establishing the meaningfulness of experimental outcomes.

\subsection{Method}

\subsubsection{Participants}

Thirty native English speaking adults participated over the internet. None of them 
A psycholinguistic investigation of predicates of personal taste

had participated in the earlier experiment.

\subsubsection{Design and materials}

Targets were two-sentence sequences as shown in ex.(6-7), with various adjectives and verbs. The subject of the first sentence was a human, referred to with a name, and the object was inanimate. The second sentence began with the pronoun 'it'. Thus, the pronoun is not ambiguous between the preceding subject and object. The question that participants answered after each target, ex.(8), disambiguates that the pronoun refers to the object and not the event of painting or hanging, for example.

We manipulated (i) the verb in the first sentence (Agent-Patient, ExperiencerTheme) and (ii) the adjective in the second sentence (PPT, non-PPT multidimensional). ${ }^{4}$ We strove to design the targets so that the experience or action of the subject of the first sentence was relevant (and felicitous) for both the PPTs and the multidimensional adjectives in the second sentence. ${ }^{5}$ Each participant saw eight targets with PPTs and eight with multidimensional adjectives, and half of these were with Agent-Patient verbs and half with Experiencer-Theme verbs. Ex.(6-8) provides an example item.

(6) a. First sentence (Experiencer-Theme verb): Lisa saw the painting. b. First sentence (Agent-Patient verb): Lisa hung the painting.

(7) a. Second sentence: It was astounding.

b. Second sentence: It was mediocre.

(8) Whose opinion is it that the painting was \{astounding / mediocre\}? Lisa's / Narrator's

\subsubsection{Procedure}

As in our earlier study, participants read excerpts (ex.6-7) and were told to imagine they were reading extracts from novels. ${ }^{6}$ For each 'story snippet',

\footnotetext{
${ }^{4} \mathrm{We}$ also tested non-judge-dependent nongradable adjectives (e.g. it was leather/free/wooden), but they are not relevant for the subjectivity-/judge-based research questions discussed here.

${ }^{5}$ Our items were designed so that the subject's experience (e.g. seeing the painting) and action (e.g. hanging the painting) could be construed as relevant for the PPTs and multidimensionals in the second sentence. Thus, we avoided situations where someone can only see an object (only has a visual experience) but the adjective makes reference to smell (olfactory experience), for example.

${ }^{6}$ Prior work by Kaiser (2015) found that processing of perspective-sensitive and subjective expressions can be modulated by the genre of the text (e.g. fiction vs. emails). In the current study
} 
participants were asked multiple-choice questions like ex.(8), which indicated who participants interpret as the judge of the PPT or multidimensional adjective. Participants could only choose one of the two answer choices (preceding subject or narrator, as exemplified in (8)); selection of both answer choices was not possible.

It is worth noting that this task is admittedly very explicit: We directly ask people about whose opinion something is. One might wonder if the explicitness of the task distorts or biases people's responses in an unnatural way. Our prior work leads us to believe that people's responses are unlikely to be distorted. To see why, we briefly review the earlier work:

Kaiser (2015) investigated readers' sensitivity to linguistic expressions hypothesized to be indicators of perspective shift away from the narrator into the perspective of a character (free indirect discourse), using sentences like (9-10). The research focused on epithet-type expressions (e.g. that ignorant jerk, poor girl) and epistemic adverbs (e.g. probably, definitely), as these have been claimed by literary narratologists to be indicators of free indirect discourse and perspective shift (McHale 1978, see also Banfield 1973; Fludernik 1993). Kaiser (2015) tested whether participants would interpret the epithet (ex.9b) or epistemic adverbial (ex.10b) as signaling a shift to the character's point-of-view, compared to a 'plain' version of the same sentence without an epithet or epistemic adverbial (ex.9a,10a). The items were designed so that interpretation of the pronoun in the second clause could be used to detect perspective shift: If a comprehender interprets 'that ignorant jerk' as being Arthur's thoughts about Eric (i.e., Arthur=judge), then 'he' refers to the preceding object Eric in ex.(9b). In the 'plain', non-epithet version of the same sentence (ex.9a), the pronoun 'he' is predicted to be more ambiguous between Arthur and Eric. Similarly, comprehenders' interpretations of the pronouns in the items with epistemic adverbs indicates whether people interpret the adverb as signaling a shift to the character's perspective.

(9) a. Arthur hollered at Eric at the restaurant. He didn't care about using foul language in a room full of people.

b. Arthur hollered at Eric at the restaurant. That ignorant jerk; he didn't care about using foul language in a room full of people.

(10) a. Hannah glanced at Ruth across the lecture hall. She wasn't paying attention to the monotone professor's lecture about chlorophyll.

b. Hannah glanced at Ruth across the lecture hall. She definitely wasn't paying attention to the monotone professor's lecture about chlorophyll

(as well as our earlier study), the stimuli were clearly presented as excerpts from fictional narratives. 
A psycholinguistic investigation of predicates of personal taste

(11) a. Who didn't care about using foul language? Arthur 123456 Eric b. Who wasn't paying attention? Hannah 1223456 Ruth

After each target, participants were saw a question like (11), which probes the referent of the pronoun. The questions used a six-point scale where the two endpoints were the two possible antecedents. The questions allowed us to probe for shifts in perspective without explicitly asking participants whose perspective a particular expression conveys. If someone shows a preference to interpret a pronoun as referring to the preceding object, this indicates a perspective shift.

In addition, the same experiment also contained an explicit point-of-view task that participants completed at the very end, after they had answered the questions about all targets and all fillers. In this second task, participants saw only the target items, with the second sentence now underlined, and were asked to indicate whose voice or point-of-view/opinion is being expressed in the underlined sentence. The choices were the subject of the sentence, the object of the sentence or the narrator. This explicit task is similar to what is used in the experiment described in the present paper. Crucially, Kaiser (2015) found that the pronounbased task that implicitly probes perspectival processing and the explicit 'whose perspective' task yielded the same kind of results: Both show that naive participants are indeed sensitive to epithets and epistemic adverbials signaling perspective shift.

Based on this earlier work, we assume that using an explicit 'whose opinion' task is not expected to distort people's responses in unexpected or unnatural ways.

\subsection{Predictions}

The predictions parallel those of our earlier experiment: If a (pragmatically and semantically relevant) referent mentioned in prior discourse as a subject-position experiencer is especially well-suited for being the judge of a subsequent PPT, then the ExpThe_PPT condition is predicted to yield more subject-opinion responses than the AgPat_PPT condition. But with multidimensional adjectives, the TheExp_MultiDim and the AgPat_Multidim conditions are predicted to yield similar proportions of subject-opinion responses: If these adjectives do not involve experiencer judges, people's answers to the 'whose opinion' question (ex.8) should not depend on whether the preceding subject is an experiencer.

Our predictions hinge on the assumption that an Experiencer-Theme verb in one sentence can provide an experiencer argument for an adjective in a subsequent sentence that seeks an experiencer judge. From a purely sentencelevel syntactic/semantic perspective, the idea that a referent's thematic role 'lingers' across sentences and influences the interpretation of a subsequent sentence may strike some as unusual. However, existing work on cross-sentential 
reference resolution has found strong evidence of thematic roles guiding pronoun resolution (and discourse-level processing more generally) across sentence boundaries. In an early study, Stevenson, Crawley \& Kleinman (1990) compared verbs with different thematic roles, including Agent-Patient verbs (e.g. hit) and Stimulus-Experiencer verbs (e.g admire), and found significant effects of thematic role on people's interpretation preferences for a pronoun in a subsequent sentence (see also Kehler \& Rohde 2013 for more recent discussion in terms of coherence relations). In light of these findings, as well as claims that the judge could be conceptualized (at least in some cases) as a null, referential pro that picks out a salient referent in the discourse model (e.g. Stephenson 2007), it does not seem unreasonable to consider the possibility that a referent's thematic role in prior discourse (and the associated semantic and pragmatic meanings) can influence how likely that referent is to be interpreted as the judge of a PPT.

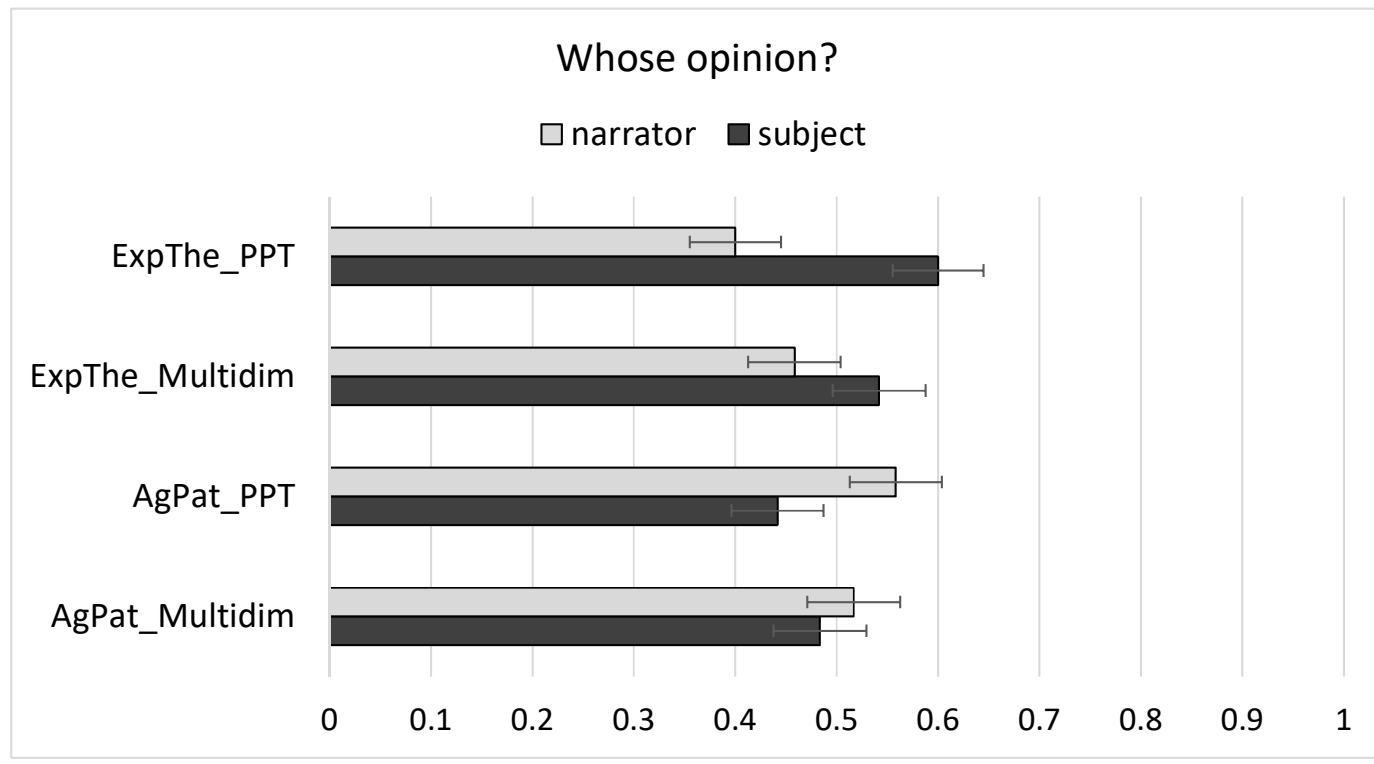

Figure 2 For sentences with unambiguous pronouns and inanimate objects: The proportion of 'subject's opinion' and 'narrator's opinion' responses to the question 'Whose opinion is it that the [object] is [adjective]? (Error bars show +/- 1 SE.) 
A psycholinguistic investigation of predicates of personal taste

\section{Results}

Figure 2 shows the proportion of 'subject's opinion' and 'narrator's opinion' responses in the four conditions (i.e., when asked 'Whose opinion is it that the [object] person was [adjective]?', how often did participants choose the preceding subject or the narrator?) Visually, it's clear that the proportion of 'subject's opinion' responses is highest in the ExpThe_PPT condition, and in particular that this is higher than in the AgPat_PPT condition. To test whether this is statistically significant, we analyzed the proportion of 'subject's opinion' responses (which is the inverse of the proportion of 'narrator's opinion' responses) using logistic mixed-effects regression models (lmer, R, R Core Team 2013). The regression models included the maximal random effects structure supported by the data, determined by building the maximal possible random effects structure (all possible random slopes for by-subject and by-item intercepts) and then simplifying if necessary for convergence.

Statistical analyses of the proportion of 'subject's opinion' responses reveal a marginal effect of verb type $(\mathrm{z}=1.734, \mathrm{p}<.09$; slightly more subject-opinion responses with Experiencer-Theme verbs than Agent-Patient verbs), no main effect of adjective type, and crucially a significant verb-type by adjective-type interaction $(\mathrm{z}=2.273, \mathrm{p}<.03)$.

Planned comparisons show that (i) with PPTs, Experiencer-Theme verbs result in a significantly higher rate of subject-opinion responses than AgentPatient verbs $(\mathrm{z}=4.154, \mathrm{p}<.001)$, but (ii) with non-PPT multidimensional adjectives, both verb types yield comparable rates of subject-opinion responses $(\mathrm{z}=1.055, \mathrm{p}>.2$, i.e. no significant difference). This replicates what we found in our earlier study: Whereas verb type matters with PPTs, it has no significant effect in the case of multidimensional non-PPT adjectives.

\subsection{Comparison of the current experiment to our earlier experiment}

Although both studies found the same kind of verb type-by-adjective type interaction in the predicted direction, one might wonder if the baseline rate of subject vs. narrator opinion choices differs significantly across the two experiments. Looking at Figures 1 and 2 might lead one to think that perhaps the first study yielded a stronger overall subject opinion preference than the current study. However, it is important to remember that participants were choosing from among three options in our earlier study (subject's opinion, object's opinion or narrator's opinion), whereas in the current study they only had two choices (subject's opinion or narrator's option). Thus, the graphs are not fully comparable. If we nevertheless run statistical analyses comparing the proportion of 'subject's opinion' responses in the two studies (i.e., if we include 'experiment' as a factor 
in the regression), we find no significant differences between the earlier study and the current study (i.e., no main effect of experiment, $p>0.16$ ) and we also find no interactions involving the factor 'experiment.' Thus, both studies yield the same pattern of results with no reliable differences.

\section{Discussion}

The two experiments discussed in this paper provide converging experimental evidence in support of the claim that the class of adjectives known as predicates of personal taste (PPTs, e.g. tasty, fun) entail experiencer judges while the judges of merely multidimensional non-PPT adjectives (e.g. healthy, smart) do not need to be experiencers (e.g. McNally \& Stojanovic 2017; Bylinina 2014). The new experiment presented in this paper builds on our earlier work (Kaiser \& Herron Lee to appear) but used stimuli that avoided potential concerns about pronoun interpretation confounding or biasing the judge interpretation effects.

Put together, the finding that both experiments found significant verb effects with PPTs (indicating a preference for judges to be experiencers) but not with non-PPT multidimensional adjectives shows that our findings are replicable, not reducible to pronoun resolution effects (recall that the pronouns in the second experiment could only refer to the preceding object in all conditions, not the subject), and not restricted to one type of coherence relation configuration (see below). Broadly speaking, our data support the idea that the specific 'type' of subjectivity associated with PPTs and non-PPT multidimensional adjectives is different: Although both adjective types are judge-dependent and subjective, only PPTs appear to exhibit a significant association between experiencers and judges.

One question that comes up is whether we could explain these results entirely based on people's general reasoning/inferencing about the coherence relations between sentences (e.g. Hobbs 1979, Kehler 2002), without having to make any reference to the idea that PPTs are sensitive to experiencer judges. A detailed answer to the question of how subjective adjective processing is guided by coherence relations (e.g. explanation relations or cause-effect relations) is beyond the scope of this paper. Coherence relations are known to have deep and farranging effects of language processing, and surely they also guide the processing of the kinds of sentence sequences that we tested. However, we would like to suggest that the process of comprehenders reasoning and drawing inferences about the likely relations between the events / states / opinions conveyed by sentences is presumably guided by the distinction that some types of subjective adjectives, but not all, entail experiencers. Under this view, the difference between PPTs and non-PPT multidimensional adjectives (i.e., whether the judge is an experiencer or not) is one part of a larger process of creating a coherent mental representation of the discourse. 
A psycholinguistic investigation of predicates of personal taste

It is worth noting that there does not appear to be any straightforward, predictable 'low-level' link in our studies between a specific coherence relation and a specific judge interpretation. For example, cause-effect relations as well as explanation relations occur in multiple conditions and can be associated with 'subject's opinion' as well as 'narrator's opinion' interpretations. Based on our results so far, it seems reasonable to conclude that the adjective-type distinction (i.e., subjective adjectives that involve experiencer judges vs. those that do not) feeds into - or forms part of - the inferencing and reasoning processes that guides discourse-level processing (Hobbs 1979; Kehler 2002), but cannot be 'explained away' by it. More generally, the way in which coherence relations relate to and guide the interpretation of subjective adjectives is an intriguing question for future work.

Another direction for future work concerns the classification of adjectives as PPTs or non-PPT multidimensional. So far we have relied largely on adjectives and diagnostics discussed in prior work and restricted ourselves to a subset of (fairly) well-understood adjectives. In future work, we plan to conduct analyses that cluster items based on people's responses to the 'whose opinion' questions (e.g. using Conditional Inference Trees, Hothorn \& Zeileis 2015; Hothorn et al. 2006), to see how the emerging clusters compare to the predicted effects of existing verb- and adjective-type classifications (cf. Djärv \& Bakovcin 2017).

\section{References}

Ambridge, Ben, Amy Bidgood, Julian M. Pine, Caroline F. Rowland and Daniel Freudenthal. 2016. Is passive syntax semantically constrained? Evidence from adult grammaticality judgment and comprehension studies. Cognitive Science 40(6), 1435-1459.

Banfield, Ann. 1973. Narrative style and the grammar of direct and indirect speech. Foundations of Language 10(1), 1-39.

Bylinina, Lisa. 2014. The Grammar of Standards. LOT Dissertation Series.

Chafe, Wallace L. 1976. Givenness, contrastiveness, definiteness, subjects, topics, and point of view. In Charles N. Li (ed.), Subject and Topic, 25-55. New York: Academic Press.

Crawley, Rosalind \& Rosemary Stevenson. 1990. Reference in single sentences and in texts. Journal of Psycholinguistic Research, 19(3), 191-210.

Crawley, Rosalind A., Rosemary J. Stevenson \& David Kleinman. 1990. The use of heuristic strategies in the interpretation of pronouns. Journal of Psycholinguistic Research, 19, 245-264.

Djärv, Kajsa and Hezekiah, A. Bacovcin. 2017. Prosodic Effects on Factive Presupposition Projection. Presented at the 27th Annual Meeting of Semantics and Linguistic Theory (SALT), University of Maryland. 
Fludernik, Monika. 1993. The Fictions of Language and the Languages of Fiction: The Linguistic Representation of Speech and Consciousness. London: Routledge.

Harris, Jesse A. and Christopher Potts. 2009. Perspective-shifting with appositives and expressives. Linguistics \& Philosophy 32(6), 523-552.

Hobbs, Jerry R. 1979. Coherence and coreference. Cognitive Science, 3, 67-90.

Hothorn, Torsten and Achim Zeileis. 2015. Partykit: A modular toolkit for recursive partytioning in R. Journal of Machine Learning Research,16, 39053909.

Hothorn, Torsten, Kurt Hornik, and Achom Zeileis. 2006. Unbiased recursive partitioning: A conditional inference framework. Journal of Computational and Graphical Statistics 15, 651-674.

Jarvis, W. Blair G. and Richard. E. Petty. 1996. The need to evaluate. Journal of Personality and Social Psychology, 70(1), 172-194.

Kaiser, Elsi. 2015. Perspective-shifting and free indirect discourse: Experimental investigations. Semantics and Linguistic Theory (SALT) 25: 346-372.

Kaiser, Elsi \& Jamie Herron Lee. To appear. Predicates of personal taste and multidimensional adjectives: An experimental investigation. To appear in 35th West Coast Conference on Formal Linguistics (WCCFL 35). Cascadilla Press.

Kamp, Hans. 1975. Two theories about adjectives. In E. L. Keenan (Ed.), Formal semantics for natural language, 123-155. Cambridge: Cambridge University Press.

Kehler, Andrew. 2002. Coherence, Reference, and the Theory of Grammar. Stanford, CA: CSLI Publications.

Kehler, Andrew \& Hannah Rohde. 2013. A Probabilistic reconciliation of coherence-driven and Centering-driven theories of pronoun interpretation. Theoretical Linguistics, 39, 1-37.

Kennedy, Christopher and Malte Willer. 2016. Subjective attitudes and counterstance contingency. Semantics and Linguistic Theory (SALT) 26: 913933.

Kennedy, Christopher. 2013. Two Sources of Subjectivity: Qualitative Assessment and Dimensional Uncertainty. Inquiry 56 (2-3), 258-277.

Klein, Ewan. 1980. A semantics for positive and comparative adjectives. Linguistics and Philosophy 4, 1-45.

Lasersohn, Peter. 2005. Context dependence, disagreement, and predicates of personal taste. Linguistics and Philosophy 28 (6), 643-86.

McHale, Brian. 1978. Free indirect discourse: A survey of recent accounts. PTL: A Journal for Descriptive Poetics and Theory of Literature 3, 249-78.

McNally, Louise and Isidora Stojanovic. 2017. Aesthetic adjectives. In James Young (ed.), The Semantics of Aesthetic Judgments, 17-37. Oxford: Oxford University Press.. 
A psycholinguistic investigation of predicates of personal taste

Ninan, Dilip. 2014. Taste predicates and the acquaintance inference. Semantics and Linguistic Theory (SALT) 24, 290-309.

Open Science Collaboration. 2015. Estimating the reproducibility of psychological science, Science 28 Aug 2015: 349 (6251), aac4716.

Patel-Grosz, Pritty. 2012. (anti-)Locality at the Interfaces. Cambridge, Massachusetts: MIT PhD dissertation.

Pearson, Hazel. 2013. A judge-free semantics for predicates of personal taste. Journal of Semantics 30(1), 103-154.

Potts, Christopher. 2007. The expressive dimension. Theoretical Linguistics 33 (2), 165-98.

$\mathrm{R}$ Core Team. 2013. $R$ : A language and environment for statistical computing. $\mathrm{R}$ Foundation for Statistical Computing, Vienna, Austria. URL http://www.Rproject.org/.

Sæbø, Kjell Johan. 2009. Judgment ascriptions. Linguistics and Philosophy, 32, 327- 352.

Sassoon, Galit W. 2013. A typology of multidimensional adjectives. Journal of Semantics 30, 335-380

Stephenson, Tamina. 2007. Judge dependence, epistemic modals, and predicates of personal taste. Linguistics and Philosophy 30 (4), 487-525.

Elsi Kaiser

Department of Linguistics

University of Southern California

Los Angeles, CA 90089

emkaiser@usc.edu
Jamie Herron Lee

Department of Linguistics

University of Southern California

Los Angeles, CA 90089

jherronlee@gmail.com 